\title{
ANALYSIS OF THE WALKINSHAW DIFFERENCE RESONANCE
}

In preparation for the Aladdin experiments, I will give an analytic treatment of the Walkinshaw difference resonance. The treatment nearly parallels that in LS-131 for the third-integral resonance.

\section{Analysis of the Resonance}

The Hamiltonian in the neighborhood of the Walkinshaw resonance

$$
\nu_{x}-2 \nu_{y}=m
$$

can be written in terms of angle-action variables in the form

$$
\begin{gathered}
h=\nu_{x} J_{x}+\nu_{y} J_{y}+s\left(2 J_{x}\right)^{1 / 2}\left(2 J_{y}\right) \sin \left(\gamma_{x}-2 \gamma_{y}-m \theta+\zeta\right) \\
\quad+a J_{x}^{2}+2 b J_{x} J_{y}+c J_{y}^{2} .
\end{gathered}
$$

We first transform to resonant coordinates via the generating function

$$
F\left(J_{1}, J_{2}, \gamma_{x}, \gamma_{y}, \theta\right)=J_{1}\left(\gamma_{x}-2 \gamma_{y}-m \theta+\zeta\right)+J_{2} \gamma_{y},
$$

which gives

$$
\begin{aligned}
& \gamma_{1}=\gamma_{x}-2 \gamma_{y}-m \theta+\zeta, \quad \gamma_{2}=\gamma_{y}, \\
& J_{x}=J_{1}, \quad J_{y}=J_{2}-2 J_{1}, \\
& J_{1}=J_{x}, \quad J_{2}=J_{y}+2 J_{x} .
\end{aligned}
$$




\section{DISCLAIMER}

Portions of this document may be illegible in electronic image products. Images are produced from the best available original document. 


\section{DISCLAIMER}

This report was prepared as an account of work sponsored by an agency of the United States Government. Neither the United States Government nor any agency thereof, nor any of their employees, makes any warranty, express or implied, or assumes any legal liability or responsibility for the accuracy, completeness, or usefulness of any information, apparatus, product, or process disclosed, or represents that its use would not infringe privately owned rights. Reference herein to any specific commercial product, process, or service by trade name, trademark, manufacturer, or otherwise does not necessarily constitute or imply its endorsement, recommendation, or favoring by the United States Government or any agency thereof. The views and opinions of authors expressed herein do not necessarily state or reflect those of the United States Government or any agency thereof. 
The resonant hamiltonian is

$$
\begin{aligned}
h_{\mathrm{w}}= & \varepsilon J_{1}+\nu_{\mathrm{y}} \mathrm{J}_{2}+2 \mathrm{~S}\left(2 \mathrm{~J}_{1}\right)^{1 / 2}\left(\mathrm{~J}_{2}-2 \mathrm{~J}_{1}\right) \sin \gamma_{1}+\mathrm{aJ}_{1}{ }^{2}+2 \mathrm{bJ}_{1}\left(\mathrm{~J}_{2}-2 \mathrm{~J}_{1}\right) \\
& +c\left(\mathrm{~J}_{2}-2 \mathrm{~J}_{1}\right)^{2},
\end{aligned}
$$

where

$$
\varepsilon=\nu_{x}-2 \nu_{y}-m
$$

We see that $J_{2}$ is a constant of the motion. Note also that the motion is required by $\mathrm{Eq}$. (1.5) to lie within the circle $2 \mathrm{~J}_{1}<\mathrm{J}_{2}$ in the $\mathrm{J}_{1}, \gamma_{1}$-phase plane.

$$
\begin{aligned}
& \text { In rectangular coordinates } \\
& Q=\left(2 \mathrm{~J}_{1}\right)^{1 / 2} \sin \gamma_{1}, \quad P=\left(2 \mathrm{~J}_{1}\right)^{1 / 2} \cos \gamma_{1},
\end{aligned}
$$

the hamiltonian is

$$
\begin{aligned}
h_{w}=\frac{1}{2} \varepsilon & \left(P^{2}+Q^{2}\right)+2 S Q\left(J_{2}-P^{2}-Q^{2}\right)+\frac{1}{4} a\left(P^{2}+Q^{2}\right)^{2}+b\left(P^{2}+Q^{2}\right)\left(J_{2}-P^{2}-Q^{2}\right) \\
& +c\left(J_{2}-P^{2}-Q^{2}\right)^{2}+\nu_{y} J_{2}
\end{aligned}
$$

For the value of $h_{w}$ corresponding to the limiting circle $J_{2}-$ $2 \mathrm{~J}_{1}=0$, Eq. (1.10) factors into the product of two circles:

$$
\left(J_{2}-P^{2}-Q^{2}\right)\left[2 S Q+b\left(P^{2}+Q^{2}\right)+c\left(J_{2}-P^{2}-Q^{2}\right)-\frac{1}{2} \varepsilon-\frac{1}{4} a J_{2}-\frac{1}{4} a\left(P^{2}+Q^{2}\right)\right]=0 .
$$


The first factor is the limiting circle, and the second is the circle

$$
P^{2}+\left(Q+\frac{S}{A}\right)^{2}=\frac{B}{A}+\frac{S^{2}}{A^{2}},
$$

where

$$
A=b-c-\frac{1}{4} a \quad, B=\frac{1}{2} \varepsilon-\left(c-\frac{1}{4} a\right) J_{2}
$$

The circles intersect (if at all) in the points

$$
Q_{0}=\left(B-A J_{2}\right) / 2 S, P_{0}= \pm\left(J_{2}-Q_{0}^{2}\right)^{1 / 2}
$$

There are two cases:

a) $\left|Q_{0}\right|>J_{2}{ }^{1 / 2}$ - Circles do not intersect. (See Fig. 1.)

b) $\left|\mathrm{Q}_{0}\right|<\mathrm{J}_{\mathrm{a}}{ }^{1 / 2}$. Circles intersect. (See Fig. 2.) The elliptic fixed points occur at the points.

$$
\mathrm{Q}_{10 \pm}=\frac{\varepsilon}{12 \mathrm{~S}}\left\{1 \pm\left[1+\frac{48 \mathrm{~S}^{2} \mathrm{~J}_{2}}{\varepsilon^{2}}\right]^{1 / 2}\right\}
$$

if we neglect the higher-order frequency shifting terms. The first-order correction for frequency-shift terms is

$$
\mathrm{Q}_{1 \pm}=\mathrm{Q}_{10 \pm} \pm \frac{\varepsilon \Delta K}{\left[\varepsilon^{2}+48 \mathrm{~S}^{2} \mathrm{~J}_{2}\right]^{1 / 2}}
$$

where

$$
\Delta \mathrm{K}=(2 \mathrm{~b}-4 \mathrm{c}) \mathrm{J}_{2} \mathrm{Q}_{10 \pm}+(\mathrm{a}+4 \mathrm{c}-4 \mathrm{~b}) \mathrm{Q}_{10 \pm}{ }^{3}
$$


It is convenient, both experimentally and theoretically to consider motions in which the $y$-amplitude is initially very small and the $x$-amplitude is finite. In that case, $J_{2}=x_{0}{ }^{2}$, where $x_{0}$ is the initial $x$-amplitude. The conditions above become
a) (Fig. 1) $x_{0}<\left|\left[\varepsilon+(a-2 b) x_{0}^{2}\right] / 4 S\right|$,
b) (Fig. 2) $x_{0}>\left|\left[\varepsilon+(a-2 b) x_{0}^{2}\right] / 4 S\right|$.

The term $(a-2 b) x_{0}{ }^{2}$ is a higher order correction due to amplitude-dependent tune shifts. From Eq $(1.4)$, we see that the condition that y remains small is that the phase point $Q, P$ remains near the limiting circle. Therefore, in case a), there is no coupling. A small initial y-amplitude remains small. In case $b$ ), the phase point cannot remain near the limiting circle. Thus if $x$ exceeds the threshold value given by Eq. (1.18), the $x$ and $y$ motions are coupled. the maximum y-amplitude occurs where the circular arc crosses the Qaxis, $i . e .$, at $(t) Q_{2}$, where

$$
Q_{2}=\left[\frac{B}{A}+\frac{S^{2}}{A^{2}}\right]^{1 / 2}-\left|\frac{S}{A}\right|,
$$

and $(t)$ is the sign of $\mathrm{S} / \mathrm{A}$.

The maximum $y$-amplitude is

$$
\begin{aligned}
y_{\max }{ }^{2} & =2\left(\mathrm{x}_{0}{ }^{2}-\mathrm{Q}_{2}{ }^{2}\right) \\
& =2 \mathrm{x}_{0}{ }^{2}-\frac{\varepsilon^{2}}{8 \mathrm{~s}}+\frac{\mathrm{b} \varepsilon^{3}}{128 \mathrm{~s}^{4}}+\left(\mathrm{c}-\frac{1}{4} \mathrm{a}\right) \frac{\varepsilon}{2 \mathrm{~s}^{2}}\left(\mathrm{x}_{\mathrm{o}}{ }^{2}-\frac{\varepsilon^{2}}{64 \mathrm{~s}^{2}}\right)+\cdots,
\end{aligned}
$$

where the last line gives the first order correction due to amplitudedependent tune shifts.

It is of interest to estimate the frequency of energy exchange between $x$ and $y$ motion $i n$ case $b$ ). Since the points $Q_{0}, \pm P_{0}$ are fixed points, the period for zero initial y-amplitude is infinite. For small initial $y$-amplitude, the phase point moves around the limiting circle in Fig. 2 until it arrives at the fixed point. It remains near the fixed point for a time, depending on the $y$-amplitude, and then traverses the circular arc up to the 
other fixed point. The y-motion therefore consists of a long time near zero -amplitude, punctuated by periodic increases to a maximum given by Eq. (1.20) and subsequent fall to near zero amplitude. If we neglect the terms in $a, b$, and $c$, the circular arc becomes the straight line

$$
Q=\frac{\varepsilon}{4 S}
$$

We can integrate the equation of motion for $P$ along this line, to obtain

$$
P=P_{0} \tanh \left(2 P_{0} s \theta\right)
$$

where $P_{0}$ is given by Eq. (1.14):

$$
\mathrm{P}_{0}^{2}=\mathrm{x}_{0}^{2}-\frac{\varepsilon^{2}}{16 \mathrm{~s}^{2}}
$$

The time scale for the pulse in y-amplitude is therefore

$$
\frac{\Delta \theta}{2 \pi}=\frac{1}{4 \pi \mathrm{P}_{0} \mathrm{~S}} \sim \frac{1}{4 \pi \mathrm{x}_{\mathrm{o}} \mathrm{S}} \text { revolutions. }
$$

We can calculate the frequency of motion about the fixed points $Q_{1 \pm^{*}}$ The result, for $\varepsilon=0$ and neglecting $a, b$, and $c$ is

$$
\nu_{w}=4 x_{0} \text { s oscillations/revolution. }
$$

The time scale is of the same order as that given by Eq. (1.24).

These time scales are shorter than radiation damping times, so it should be possible to see coupling phenomena. Radiation damping will damp the motion in Fig. 2 toward the fixed points, at the same time damping the value of $\mathrm{J}_{2}$, which will move the system toward the non-resonant case, unless $\varepsilon=0$. 


\section{Connection with the Real Ring}

We follow the same analysis as in LS-131 through Eq. [2.7]. (I wil1 use square bracket to indicate equation numbers from LS-131.) We substitute from Eq. [2.3] in Eq. [2.6], to obtain, in place of Eq. [2.8],

$$
\begin{aligned}
H_{W}= & R^{-1} H_{o}\left(J_{x}, J_{y}\right)+\frac{B^{\prime \prime} \ell}{6 B \rho} \delta\left(s-s_{j}\right)\left(2 J_{x}\right)^{3 / 2} \beta_{x}^{3 / 2} \sin ^{3}\left(\gamma_{x}-\psi_{x}\right) \\
& -\frac{B^{\prime \prime} \ell}{2 B \rho} \delta\left(s-s_{j}\right) \beta_{x}{ }^{1 / 2} \beta_{y}\left(2 J_{x}\right)^{1 / 2}\left(2 J_{y}\right) \sin \left(\gamma_{x}-\psi_{x}\right) \sin ^{2}\left(\gamma_{y}-\psi_{y}\right),
\end{aligned}
$$

where

$$
\mathrm{H}_{\mathrm{o}}\left(\mathrm{J}_{\mathrm{x}}, \mathrm{J}_{\mathrm{y}}\right)=\nu_{\mathrm{x}} \mathrm{J}_{\mathrm{x}}+\nu_{\mathrm{y}} \mathrm{J}_{\mathrm{y}}+\mathrm{aJ}_{\mathrm{x}}{ }^{2}+2 \mathrm{bJ} \mathrm{J}_{\mathrm{x}} \mathrm{J}_{\mathrm{c}}+\mathrm{J}_{\mathrm{y}}^{2}
$$

We now make the substitutions [2.9] and [2.10], with the result, in place of Eq. [2.11],

$$
\begin{aligned}
\mathrm{H}_{\theta}= & \mathrm{H}_{0}\left(\mathrm{~J}_{x}, \mathrm{~J}_{y}\right)+\sum_{m=-\infty}^{\infty} \mathrm{S}_{3}\left(2 J_{x}\right)^{3 / 2}\left[-\sin \left(3 \gamma_{x}-m \theta+m s_{j} / R-3 \psi_{x j}\right)\right. \\
& \left.+3 \sin \left(\gamma_{x}-m \theta+m s_{j} / R-\psi_{x j}\right)\right] \\
-\sum_{m=-\infty}^{\infty} S_{w}\left(2 J_{x}\right)^{1 / 2}\left(2 J_{y}\right)\left[-\sin \left(\gamma_{x}+2 \gamma_{y}-m \theta+m s_{j} / R-\psi_{x j}-2 \psi_{y j}\right)\right. & \left.-\sin \left(\gamma_{x}-2 \gamma_{y}-m \theta+m s_{j} / R-\psi_{x j}+2 \psi_{y j}\right)+2 \sin \left(\gamma_{x}-m \theta+m s_{j} / R-\psi_{x j}\right)\right],
\end{aligned}
$$

where $\mathrm{S}_{3}$ is given by $\mathrm{Eq} \cdot[2.12]$, and

$$
S_{W}=\left(\frac{x^{1 / 2} \beta y^{B " \ell}}{16 \pi B p}\right)_{s=s_{j}} \text {. }
$$

After dropping or transforming away the non-resonant terms, we are left with the Hamiltonian (1.2), with $\mathrm{S}$ given by Eq. (2.4), and

$$
\zeta=m s_{j} / R-\psi_{x j}+2 \psi_{y j}
$$




\section{Transforming the Non-Resonant Terms}

The non-resonant terms in Eq. (2.3) can be transformed away by the method used in LS-131, Section 3. We introduce a generating function

where

$$
F=J_{x} \gamma_{x}+J_{y} \gamma_{y}+\left(2 J_{x}\right)^{3 / 2} \Sigma_{3}+\left(2 J_{x}\right)^{1 / 2}\left(2 J_{y}\right) \Sigma_{w}
$$

$$
\begin{aligned}
\Sigma_{3}= & \sum_{m}\left[F_{m^{\prime}}{ } \cos \left(3 \gamma_{x}-m \theta+m s_{j} / R-3 \psi_{x j}\right)+F_{m^{\prime} 31} \cos \left(\gamma_{x}-m \theta+m s_{j} / R-\psi_{x j}\right)\right], \\
\Sigma_{w}= & \sum_{m^{\prime}}\left[F_{m^{\prime}+} \cos \left(\gamma_{x}+2 \gamma_{y}-m \theta+m s_{j} / R-\psi-2 \psi_{y j}\right)\right. \\
& +F_{m^{\prime}-} \cos \left(\gamma_{x}-2 \gamma_{y}-m \theta+m s_{j} / R-\psi_{x j}+2 \psi_{y j}\right) \\
& \left.+F_{m^{\prime} w 1} \cos \left(\gamma_{x}-m \theta+m s_{j} / R-\psi_{x j}\right)\right]
\end{aligned}
$$

The transformation equations for $\mathrm{J}$ are

$$
\begin{aligned}
& \mathrm{J}_{\mathrm{x}}=\frac{\partial F}{\partial \gamma_{\mathrm{x}}}=\mathrm{J}_{\mathrm{x}}+\left(2 \mathrm{~J}_{\mathrm{x}}\right)^{3 / 2} \frac{\partial \Sigma_{3}}{\partial \gamma_{\mathrm{x}}}+\left(2 \mathrm{~J}_{\mathrm{x}}\right)^{1 / 2}\left(2 \mathrm{~J}_{\mathrm{y}}\right) \frac{\partial \Sigma_{\mathrm{w}}}{\partial \gamma_{\mathrm{x}}}, \\
& \mathrm{J}_{\mathrm{y}}=\frac{\partial F}{\partial \gamma_{\mathrm{y}}}=\mathrm{J}_{\mathrm{y}}+\left(2 \mathrm{~J}_{\mathrm{x}}\right)^{3 / 2} \frac{\partial \Sigma_{3}}{\partial \gamma_{\mathrm{y}}}+\left(2 \mathrm{~J}_{\mathrm{x}}\right)^{1 / 2}\left(2 \mathrm{~J}_{\mathrm{x}}\right) \frac{\partial \Sigma_{\mathrm{w}}}{\partial \gamma_{\mathrm{y}}}
\end{aligned}
$$

The third-order terms in $\underline{H}_{\theta}$ can be written as in $\mathrm{Eq} \cdot[3.4]$. They can be made to vanish by setting

$$
\begin{aligned}
& F_{m ! 3}=\frac{-S_{3}}{3 \nu_{x}-m^{\prime}}, \quad F_{m^{\prime} 31}=\frac{3 S_{3}}{\nu_{x}-m^{\prime}}, \\
& F_{m^{\prime}+}=\frac{S_{w}}{\nu_{x}+2 \nu_{y}-m^{\prime}}, \quad F_{m^{\prime}-}=\frac{S_{w}}{\nu_{x}-2 \nu_{y}-m^{\prime}}, \quad F_{m} w^{\prime}=\frac{-2 S_{w}}{\nu_{x}-m^{\prime}} .
\end{aligned}
$$

We do not want to transform away the resonant term, so we set $\mathrm{F}_{\mathrm{m}-}=0$, for the value $\mathrm{m}^{\prime}=\mathrm{m}$ corresponding to the resonance $(1.1)$. (In Aladdin, $m=-7$. ) 
We can now calculate the $\theta$-independent terms of fourth order

in $\underline{H}_{\theta}$. The resulting corrections to the coefficients in Eq. (2.2) are

$$
\begin{aligned}
& a_{s}=6 s_{3}^{2} \sum_{m}\left[\frac{3}{m^{1}-3 \nu_{x}}+\frac{1}{m^{1}-v_{x}} \doteq 6 s_{3}{ }^{2}\left[-\frac{1}{\delta \nu_{x}}-\frac{3}{\delta\left(3 \nu_{x}\right)}+2 \delta \nu_{x}+6 \delta\left(3 \nu_{x}\right)\right]\right. \text {, } \\
& b_{s}=3 S_{3} \sum_{m^{\prime}}^{\sum}\left(-3 F_{m^{\prime} w 1}\right)-s_{w_{m^{\prime}}} \sum_{m^{\prime}}\left[-2 F_{m^{\prime} 31}-4 F_{m^{\prime} w 1}+2 F_{m^{\prime}+}+2 F_{m^{\prime}-}\right] \\
& =\Sigma^{\prime} \cdot\left\{\frac{24 S_{3} S_{w}-8 S_{w}^{2}}{\nu_{x}-m^{\prime}}-\frac{2 S_{w}^{2}}{\nu_{x}+2 \nu_{y}-m^{2}}-\frac{2 s_{w}^{2}}{\nu_{x}-2 \nu_{y}-m^{\prime}}\right\} \\
& \doteq\left(24 S_{3} S_{w}-8 S_{w}^{2}\right)\left[\frac{1}{\delta \nu_{x}}-2 \delta \nu_{x}\right]-2 S_{w}{ }^{2}\left[\frac{1}{\delta\left(\nu_{x}+2 \nu_{y}\right)}-2 \delta\left(\nu_{x}+2 \nu_{y}\right)-2 \delta\left(\nu_{x}-2 \nu_{y}\right)\right] \\
& c_{s}=-2 S_{W_{m^{\prime}}} \sum_{m^{\prime} w^{\prime} 1^{\prime}}\left[-2 F_{m^{\prime}+}+F_{m^{\prime}-}\right]=-12 S_{w^{2}}^{2} \sum_{m^{\prime}}^{\Sigma^{\prime}}\left[\frac{4}{\nu_{x}-m^{\prime}}+\frac{1}{\nu_{x}+2 \nu_{y}-m^{\prime}}+\frac{1}{\nu_{x}-2 \nu_{y}-m^{\prime}}\right] \\
& =-2 S_{w}^{2}\left[\frac{4}{\delta \nu_{x}}+\frac{1}{\delta\left(\nu_{x}+2 \nu_{y}\right)}-4 \delta \nu_{x}-\delta\left(\nu_{x}+2 \nu_{y}\right)-\delta\left(\nu_{x}-2 \nu_{y}\right)\right]
\end{aligned}
$$

Formula (3.5) agrees with [3.7], except that there ts no missing resonant term. The term $\delta z$ is again defined as $z-m$, where $m$ is the nearest integer to $z$. 


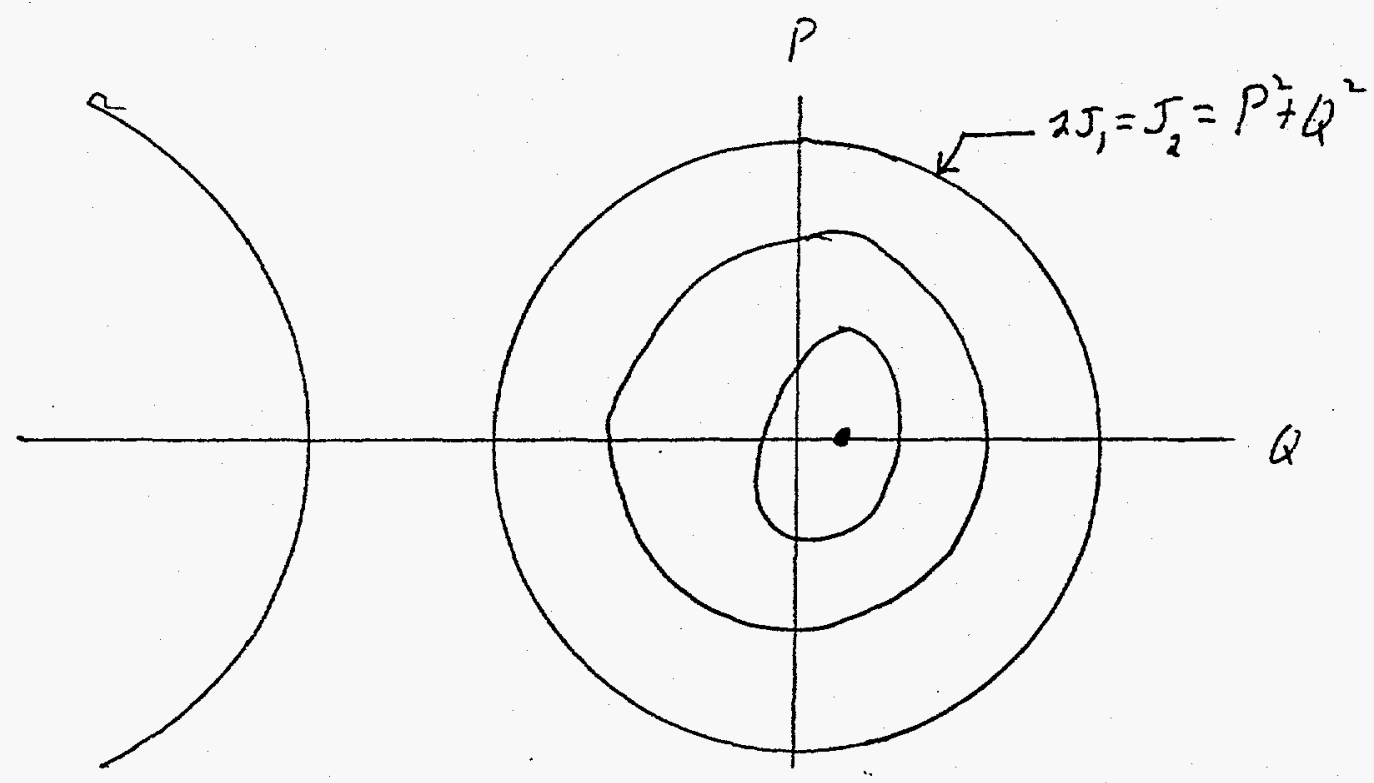

Fig. 1. Non-resonant case.

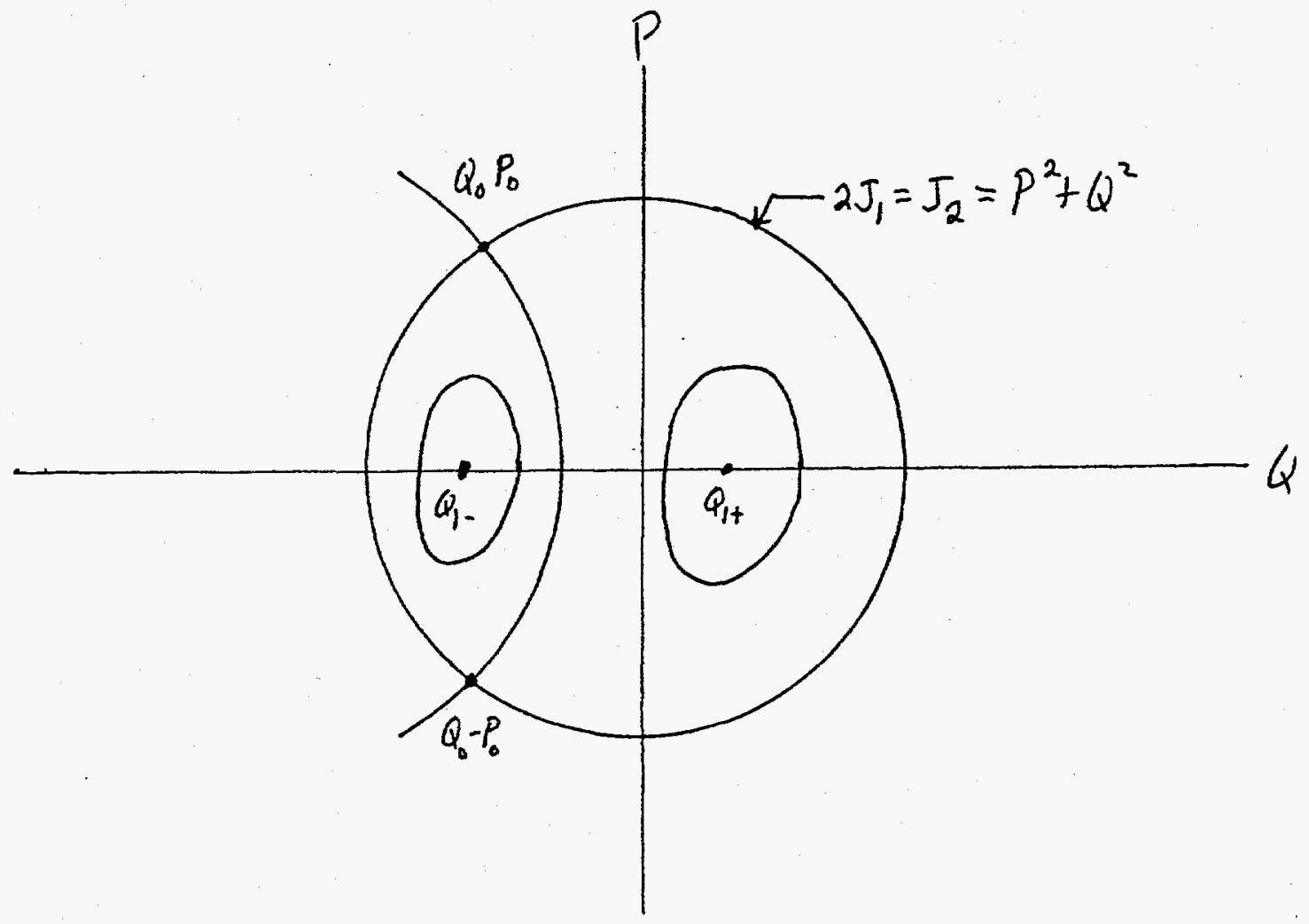

Fig. 2. Resonant Case 